\begin{tabular}{|l|l|c|c|}
\hline & & $107-111$ & Hannover 1997 \\
\hline
\end{tabular}

\title{
Ein frühweichselzeitliches Profil bei Rahden-Welle (nördliches Wiehengebirgsvorland, Nordrhein-Westfalen)
}

\author{
KLAus SKupin \& RÜDIGER STRITZKE*)
}

Pleistocene, Early Weichselian, mapping, gravel and pollen analysis

\begin{abstract}
Kurzfassung: Bei Aushubarbeiten an der Kläranlage in Rahden-Welle wurden Niederterrassenablagerungen der Kleinen Aue ergraben. Unter Auen- und Talsanden erschloß sich eine 1-2 m mächtige Silt- und Torfschicht, auf die im Liegenden vermutlich saalezeitliche Schmelzwassersande folgten. Der pollenanalytischen Bearbeitung der Torfschicht zufolge prägten vor allem Gräser sowie Kiefer und Birke das damalige Landschaftsbild. In Verbindung mit dem Nachweis erheblicher Heliophytenanteile erlaubt das pollenfloristische Material eine Einstufung in das HerningStadial der frühen Weichsel-Kaltzeit.
\end{abstract}

\section{[An Early Weichselian section near Rahden-Welle]}

\begin{abstract}
During excavations for a purification plant in Rahden-Welle lower terrace depositions of the „Kleine Aue" were found. Meltwater layers of probably Saalian age were overlain by peaty, silty and sandy depositions. Pollenanalytical investigation of the peat records the dominance of grass, pine and birch in the scenery. Together with considerable amounts of heliophytes the pollen assemblage indicates an Herning stadial age (early Weichselian) of the investigated layer.
\end{abstract}

\section{Einleitung}

Bei der geologischen Kartierung des Blattes 3517 Rahden im nördlichen Wiehengebirgsvorland wurde eine temporär aufgeschlossene Baugrube im Bereich der Kläranlage „Am Stellerdamm“ in RahdenWelle untersucht (Abb. 1). Erschlossen waren weichselzeitliche Ablagerungen der Kleinen Aue, einem Quellfluß der Aue, welche der Hunte zufließt. Der Untergrund war bis auf eine Tiefe von etwa $6 \mathrm{~m}$ großräumig aufgeschlossen.

\section{Geologie}

Den quartären Untergrund des Blattes Rahden bilden 10-30 m mächtige Sedimente des Mittel- bis Jungpleistozäns, deren Basis Schmelzwassersedimente und Geschiebemergel der Elster-Kaltzeit mar-

\footnotetext{
*) Anschrift der Verfasser: Dr. K. SkUPIN und Dr. R. STRITZKE, Geologisches Landesamt Nordrhein-Westfalen, Postfach 1080, 47710 Krefeld
}

kieren (Deutloff 1986). Im Hangenden folgen zumeist Mittelterrassen-Ablagerungen der Weser, deren Verlauf im Vorfeld des saalezeitlichen Inlandeises damals E-W gerichtet war (RoHDE1994). Im Bereich von Rahden und Wehe finden sich in Geländekuppen saalezeitliche Sedimente erhalten, die als Teil eines SE-NW gerichteten Stauchmoränenrückens, der Hiller Staffel, anzusprechen sind (Wortmann 1971, Skupin \& Speetzen i. Vorb.). Den oberflächennahen Untergrund des unmittelbaren Untersuchungsgebietes bilden zumeist fluviatile Sedimente der Weichsel-Kaltzeit und des Holozäns sowie holozäne Moor- und Anmoorbildungen. Derartigen Ablagerungen entstammt das untersuchte Profil der Kläranlage Rahden-Welle. Es läßt sich in drei Abschnitte gliedern mit Sanden und Kiesen im oberen und unteren Teil sowie kryoturbar stark verformten Silten und Torfen dazwischen (Abb. 2 und 3). Den tieferen Untergrund erschloß eine unmittelbare benachbarte Spülbohrung, die bei $17 \mathrm{~m}$ Teufe endete. Demnach folgen im Liegenden des Profils siltige Sande und sandige Kiese über Tonsteinen der Unterkreide. Die sandigen Kiese repräsentieren aufgrund ihres Gehaltes an nordischen Kristallingeschieben entweder weichselzeitliche Terrassenkörper oder saalezeitliche Rinnenfüllungen.

\section{Paläobotanik 3.1 Methodik}

Die Probenentnahme für die Pollenanalyse erfolgte engständig im Abstand von 5 bis $10 \mathrm{~cm}$. Die Pollenaufbereitung geschah nach einer Vorbehandlung mit $\mathrm{KOH}$ und 45\%iger HF nach der Acetolyse-Methode von Erdtmann. Abschließend wurden das Probenmaterial gesiebt $(10 \mu \mathrm{m})$. Die Berechnungsgrundlage für die Pollentaxa ist die Summe aller Baumpollen (BP). Das Pollendiagramm ist in sog. „Iocal pollen assemblage zones“ (LPAZ), lokale Pollenvergesellschaftungszonen unterteilt worden. Zur Artbestimmung von Betula wurden in repräsentativen Proben die Korndurchmesser ermittelt. Die Messung erfolgte von einer Wand zur gegenüberliegenden Pore. 


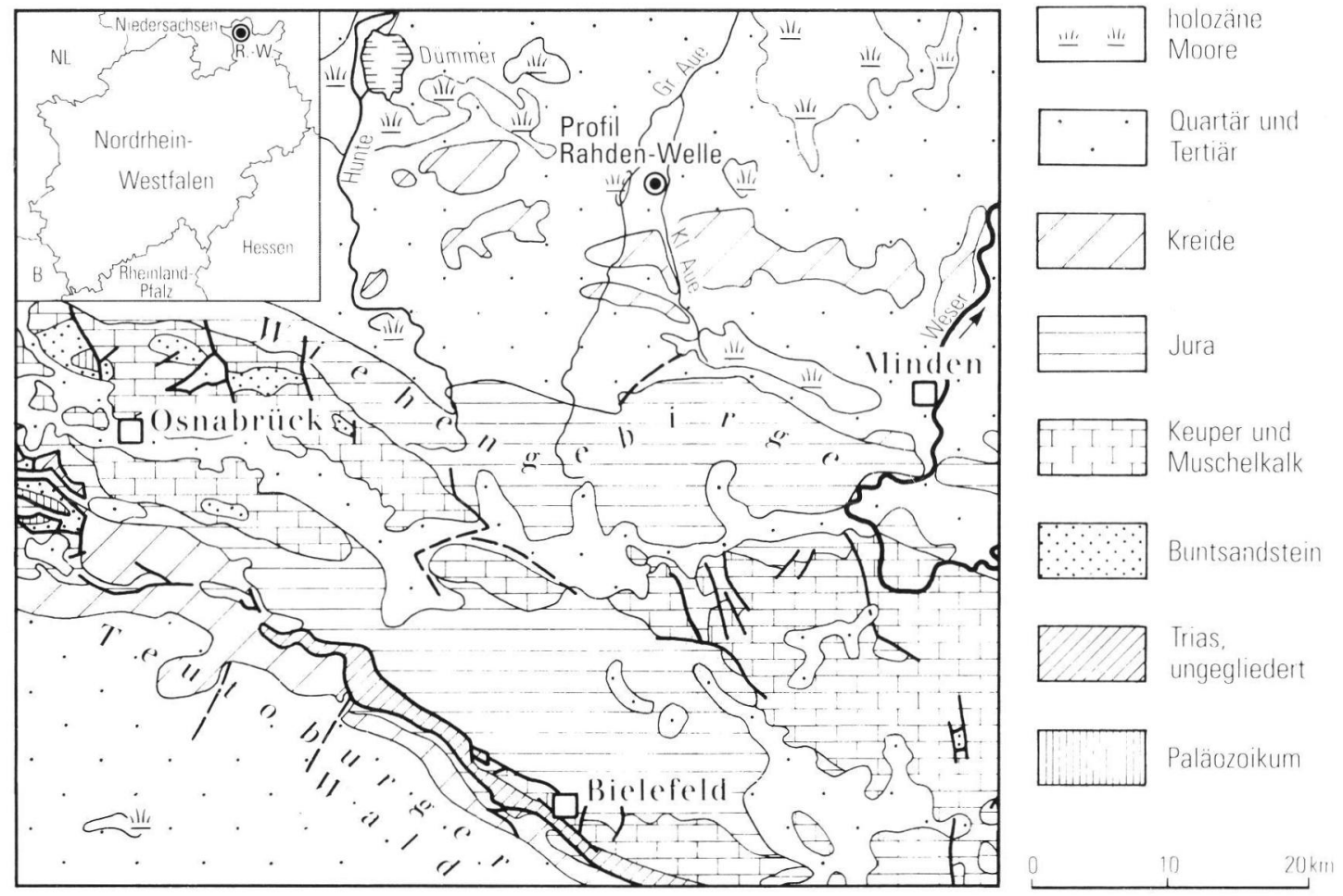

Abb. 1: Untersuchungsgebiet und Lage des Profils Rahden-Welle.

Fig.1: Study area and location of section Rabden-Welle.

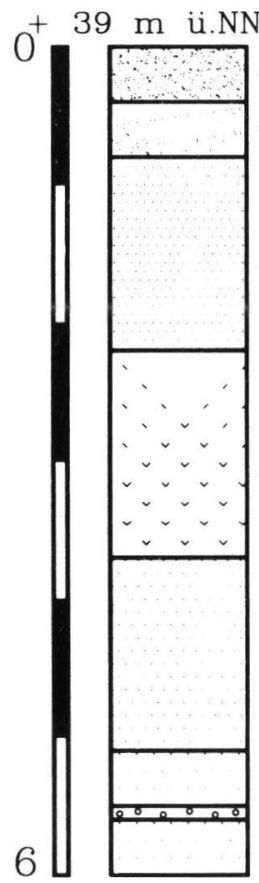

Mittelsand, stark feinsandig, schwach sultig, humos, schwarzbraun

Mittelsand, lagenweise stark feinsandig, schwach siltig. schwach grobsandig, schwach kiesig (Grob- bis Feinkies),

flaserig-wellig, gellbraun bis weibgrau

Fein- bis Mittelsand, schwach siltig - siltig, gelbbraun

bis gelbgrau, in kryoturbat vorliegenden Taschen und Nestern

z.T. humos mit Holzstücken, grau bis dunkel- oder schwarzgrau

Torf, siltig - stark siltig (= Torfmudde), schwarzbraun,

im Wechsel mit Silt, torfig - stark torfig, schwach fein-

sandig (=Siltmudde), dunkelgraubraun, nach oben und unten kryoturbat mit Sand wechsellagernd

Fein- bis Mittelsand, schwach siltig - siltig, stellenweise stark feinsandig, schwach kiesig (Fein- bis Mittelkies), hellgrau, von tropfen- und taschenförmigen Torf- und torfigen Silteinlagerungen durchsetzt

$\mathrm{m}$

Fein- bis Mittelsand, schwach siltig - siltig, schwach kiesig, grau, kryoturbat gestört Fein-, Mittel- und Gribkies, sandig, schwach siltig, graubraun bis braungrau Mittelsand, stark feinsandig, schwach kiesig, schwach siltig, kryoturbat gestort, graubraun bis rostbraun 


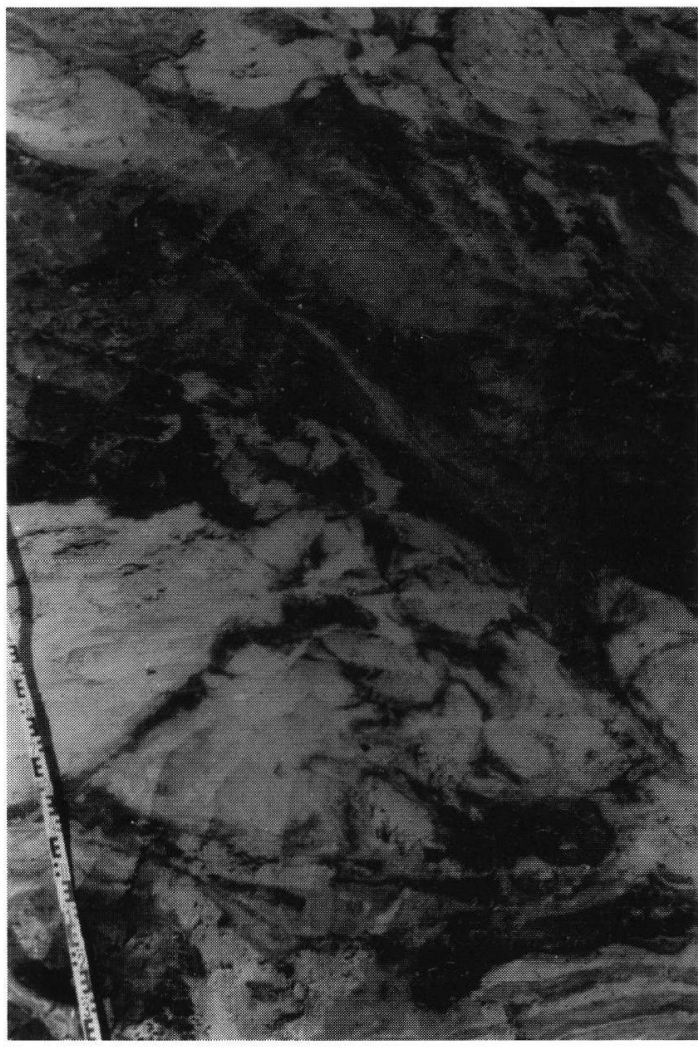

Abb.3: Siltige und torfige Ablagerungen des HerningStadials / Profil Rabden-Welle.

Fig.3: Silty and peaty depositions of the Herning stadial / Section Rabden-Welle.

\subsection{Palynologischer Befund}

Das Profil läßt sich in vier lokale Pollenvergesellschaftungszonen (LPAZ) untergliedern (Abb. 4):

\section{Zone R I}

(Pinus-Cyperaceae-Betula-Poaceae-LPAZ)

$(2,00-2,75 \mathrm{~m})$

Die unterste LPAZ wird von Kiefer (Pinus) und Birke (Betula) bestimmt, die Werte um 50 bzw. 30\% der Baumpollensumme einnehmen. Die Fichtenkurve (Picea) ist auf niedrigem Niveau $(<10 \%)$ geschlossen. Die Weide (Salix) ist markant und erreicht bei $2,35 \mathrm{~m}$ ein lokales Maximum (22\%). Die wenigen Pollen thermophiler Gehölze (Eiche, Ulme, Hainbuche) sind sicherlich eingeweht. Unter den Pollen krautiger Pflanzen herrschen die der Gräser vor; dabei dominieren die Sauergräser (Cyperaceae) bis 107\% der Baumpollensumme. Die Quoten der Süßgräser (Poaceae) schwanken um 20\%. Schließlich fallen hohe Torfmoos-(Sphagnum-)Werte auf (um 25\%). Die Betula-Werte gipfeln bei $20 \mu \mathrm{m}$ (Abb. 5a). Aufgrund der Dominanz kleinwüchsiger Formen ist ein Betula nana-Vorkommen anzunehmen.

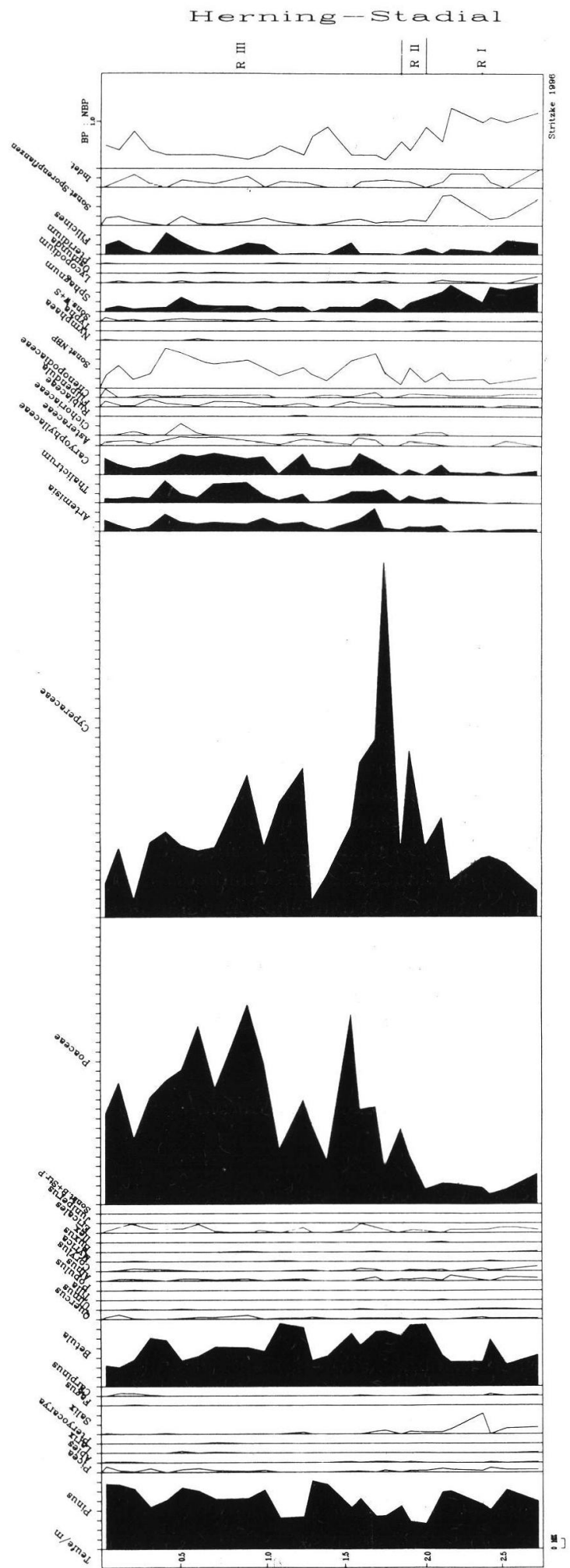

Abb.4: Pollendiagramm des Profils Rabden-Welle. Fig. 4: Pollen diagram of the section Rabden-Welle. 
Probe 1: $1.1 \mathrm{~cm}$
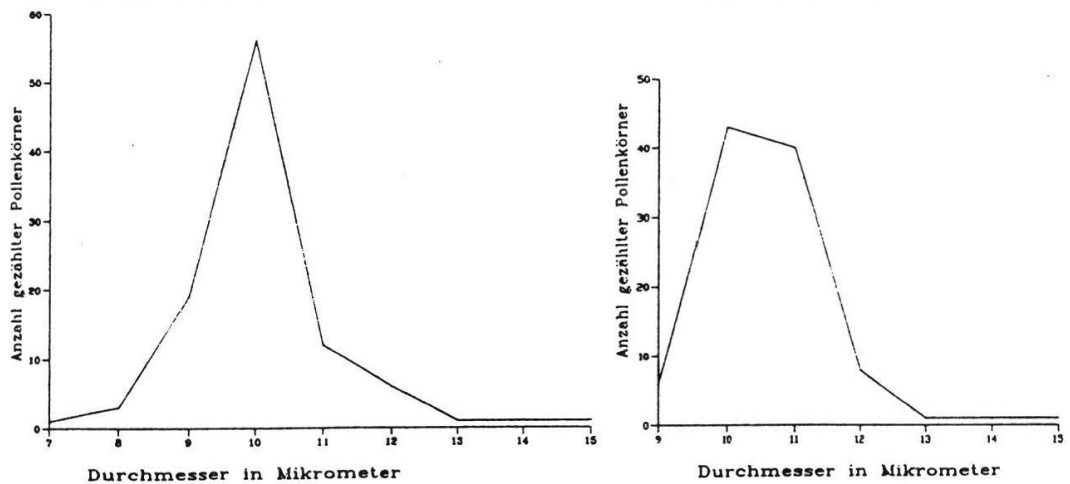

Abb. 5: Verteilung der Birkenpollen-Durchmesser in drei repräsentativen Proben des Profils Rabden-Welle.

Fig.5: Distribution of birch pollen diameters within three representative sampels of section Rabden-Welle.

\section{Zone R II}

(Cyperaceae-Poaceae-Betula-Pinus-LPAZ)

$(1,90-1,85 \mathrm{~m})$

In dieser geringmächtigen Übergangszone nehmen die Pollen krautiger Pflanzen gegenüber denen der Gehölze an Bedeutung zu. Die Kiefer ist deutlich rückläufig und wird vorübergehend von der Birke überflügelt. Andere Gehölzpollen treten nur sporadisch in sehr geringen Quoten auf; sie dürften sämtlich eingeweht sein. Die Kurve der Süßgräser schneidet in dieser Zone die der Sauergräser. Sauergräser werden im Hangenden das dominierende Florenelement. Andere Nichtbaumpollen sind in geringen Mengen nachgewiesen.

Die Betula-Werte weisen Maxima bei 20 und $22 \mu \mathrm{m}$ auf (Abb. 5b). Demzufolge ist auch hier ein Betula nana-Vorkommen anzunehmen.

\section{Zone R III}

(Poaceae-Cyperaceae-Pinus-Betula-LPAZ) $(0,02-1,75 \mathrm{~m})$

Kiefer und Birke bleiben die vorherrschenden Gehölze, wobei im Verlaufe der Zone die Kiefernkurve leicht ansteigt, die der Birke abfällt. Die Fichte wird im oberen Teil der Zone wieder etwas bedeutsam; ihre Kurve ist fast geschlossen.

Die dominierenden Florenelemente aber sind die Pollen krautiger Pflanzen, vor allem die der Süßund Sauergräser. Auffallend ist darüber hinaus eine deutliche Zunahme vieler Heliophyten: Beifuß ( $A r-$ temisia), Wiesenraute (Thalictrum), Nelkengewächse (Caryophyllaceae), Korbblütler (Asteraceae). Sie erreichen Maxima bis zu 24\%. Ergänzt wird dieses Spektrum durch Pollen der Gänsefußgewächse (Chenopodiaceae), Erikagewächse (Ericaceae) und Mädesüß (Filipendula).

Die Betula-Körner messen durchschnittlich $20 \mu \mathrm{m}$
(Abb. 5c). Es dürfte sich auch hier um ein Betula-nanaVorkommen handeln.

\section{Diskussion}

Der pollenanalytische Befund dokumentiert eine baumbestandene Tundrenlandschaft mit Kiefern und Birken, die sich allmählich in eine halboffene Taigalandschaft wandelt, denn es treten im oberen Teil verstärkt Heliophyten in Erscheinung. Diese Vegetationsform wird als forest/tundra ecotone bezeichnet, eine Vegetationszone zwischen Tundra und Taiga oder dem borealen Kiefern- und Birkenwald (EDMONSPOHL 1995). Derartige Vegetationsverhältnisse finden sich wiederholt in Stadialen und Interstadialen. Da sich die beschriebene Torfschicht aber in glazigenen Sedimenten befindet, die jünger als_saalezeitlich sind, kommt nur ein weichselzeitliches Alter in Betracht. Darüber hinaus lassen sich die pollenanalytischen Gegebenheiten gut mit frühweichselzeitlichen Sequenzen anderer Gebiete Mitteleuropas konnektieren.

So beschreiben Menke \& TYNnI (1984) aus Westholstein ein eem- bis frühweichselzeitliches Profil in Rederstall. Ihre Zone WF I (Herning-Stadial) läßt sich durch ein kleines Betula-Maximum (WF Ib) untergliedern. Im darüberliegenden Abschnitt (WF Ic) nimmt der Anteil der Heliophyten deutlich zu. Diese Beobachtungen lassen sich mühelos mit den Zonen R II und R III in Rahden verknüpfen.

Ähnliche Übereinstimmungen finden sich auch mit den Oerel-Bohrungen in Niedersachsen BEHRE \& LADE (1986). Auffallend sind insbesondere Übereinstimmungen mit der Zone WF I, die dem HerningStadial zugeordnet wird.

LiTT (1990) dokumentiert aus der Grube Gröbern in Sachsen eine saale- bis weichselkaltzeitliche Abfolge. Die frühweichselzeitliche Pollenzone WF I stimmt in ihrer palynologischen Zusammensetzung 
mit der gleichnamigen Zone aus Oerel-Bohrungen weitgehend überein. Außerdem wird im höheren Teil dieser Zone ein steigender Heliophyten-Anteil (u. a. Artemisia, Chenopodiaceae, Thalictrum) beschrieben. Dieser Abschnitt läßt sich unmittelbar mit der Zone R III aus Rahden vergleichen. Auch dort steigt der Heliophytenanteil signifikant.

Aufgrund dieser eindeutigen mikrofloristischen Übereinstimmungen ist es plausibel, den untersuchten torfigen Profilabschnitt des Aufschlusses in Rahden-Welle dem Herning-Stadial der frühen Weichsel-Kaltzeit zuzuordnen.

\section{Danksagung}

Die Stadt Rahden stellte das Bodengutachten zur Kläranlage Rahden-Welle, erstellt durch das Erdbaulabor und Ingenieurbüro Schlemm, zur Auswertung zur Verfügung.

\section{Schriftenverzeichnis}

BeHRE, K.-E., \& LADE, U. (1986): Eine Folge von Eem und 4 Weichsel-Interstadialen in Oerel/Niedersachsen und ihr Vegetationsablauf..-Eiszeitalter u. Gegenwart, 36: 11-36, 12 Abb., 2 Tab., 2 Taf.; Öhringen
EdmontspoHL, A.-F. (1995): The northwest European vegetation at the beginning of the Weichselian glacial (Brorup and Odderade interstadials) - new data for northern France.-Rev. Palaeobot. Palynol., 85: 231-242, 8 Abb.; Amsterdam.

LITT, T. (1990): Pollenanalytische Untersuchungen zur Vegeationsund Klimaentwicklung während des Jungpleistozäns in den Becken von Gröbern und Grabschütz.- Altenburger naturwiss. Forsch., 5: 92-105, 5 Abb., 3 Tab; Altenburg.

MENKE, B., \& IYNNI, R. (1984): Das Eeminterglazial und das Weichselfrühglazial von Rederstall/Dithmarschen und ihre Bedeutung für die mitteleuropäische Jungpleistozän-Gliederung.Geol. Jb., A/76: 1-120, 18 Abb., 7 Tab., 9 Taf.; Hannover.

Deutloff, O. (1986): Blatt C 3914 Bielefeld.-Geol. Kt. Nordrh.Westf. 1:100.000, C 3914: 1 Kt., mit Erl. von Deutloff, O. \& Kühn-Velten, H., \& Michel, G.: 104 S., 19 Abb., 2 Tab.; Krefeld.

ROHDE, (1994): Weser und Leine am Berglandrand zur Ober- und Mittelterrassen-Zeit. - Eiszeitalter u. Gegenw., 44: 106-113, 2 Abb.; Hannover.

Skupin, K. \& SpeETzen, E. (i.Vorb.): Die Stauchmoräne von Oppenwehe bei Rahden (TK 3517 Rahden, nördliches Wiehengebirgsvorland). - Osnabrücker naturwiss. Mitt.

Wortmann, H. (1971), unter Mitarb. von Michel, G., \& ReHagen, H.W.: Erläuterungen zu Blatt 3617 Lübbecke und Blatt 3618 Hartum. - Geol. Kt. Nordrh.-Westf. 1:25 000, Erl., 3617, 3618 : 214 S., 24 Abb., 13 Tab., 3 Taf.; Krefeld.

Manuskript eingegangen am 09.07.1996 